\title{
Choroideremia associated with choroidal neovascularization treated with intravitreal bevacizumab
}

This article was published in the following Dove Press journal:

Clinical Ophthalmology

I September 2014

Number of times this article has been viewed

\author{
Neal V Palejwala \\ Andreas K Lauer \\ Richard G Weleber \\ Oregon Retinal Degeneration Center \\ (ORDC), Ophthalmic Genetics \\ Service and Retina-Vitreous Service, \\ Casey Eye Institute, Oregon Health \\ and Science University, Portland, OR, \\ USA
}

Purpose: To report a rare case of central vision loss in a patient with choroideremia.

Patients and methods: A retrospective, interventional case report.

Results: A 13-year-old male with history of choroideremia presented with subacute loss of central acuity in his left eye. Examination and diagnostic testing revealed subretinal fibrosis secondary to a choroidal neovascular membrane (CNVM). A trial of anti-vascular endothelial growth factor (VEGF) therapy with the injection of intravitreal bevacizumab was attempted. Mild improvements in acuity and anatomy were noted.

Conclusion: Choroideremia is a rare hereditary choroidal dystrophy that predominantly affects males in the first and second decades of life. Visual acuity is usually spared until later in life. CNVM is a rare manifestation of choroideremia with only a handful of case reports presented in the literature. This case is unique in that it is the first reported case that received treatment with intravitreal anti-VEGF therapy.

Keywords: anti-VEGF therapy, choroideremia, choroidal neovascular membrane, chorioretinal degeneration, hereditary choroidal dystrophy, intravitreal bevacizumab injection

\section{Introduction}

First described in 1872 by Mauthner, ${ }^{1}$ choroideremia is a hereditary choroidal dystrophy inherited as an X-linked recessive trait. ${ }^{2}$ It has an estimated prevalence of 1 in 50,000 with male predominance, although cases of symptomatic female carriers have been identified. ${ }^{3}$ Affected males typically present with impaired night vision and peripheral visual field loss in the first and second decades. Central vision is not involved until later in the disease course, generally in the fifth to seventh decade. Fundus changes commence with fine, mid-peripheral retinal pigment mottling followed by underlying choroidal atrophy. Changes slowly progress centrally with the macula being wellpreserved for many years. Histopathologic studies have shown extensive atrophy of the choroidal vasculature, retinal pigment epithelium (RPE), and photoreceptors with relative preservation of the inner retinal layers. ${ }^{4}$

Central vision loss has most commonly been reported secondary to underlying outer retinal/choroidal atrophy and is a late manifestation of the disease. Rarely have cases of early loss of visual acuity been cited. In the current case, we review an unusual cause of vision loss in choroideremia: choroidal neovascular membrane (CNVM).

\section{Case report}

A 13-year-old Caucasian male previously diagnosed at 7 years of age with choroideremia presented with subacute loss of central acuity in his left eye. The patient has been 
followed routinely with the Ophthalmic Genetics Service at Casey Eye Institute and has maintained 20/20 visual acuity bilaterally, until now. The visual acuity was 20/20 and 20/100 in his right and left eye, respectively. An afferent pupillary defect was absent. Tonometry and anterior segment examination were within normal limits. Dilated fundus examination revealed diffuse peripheral chorioretinal atrophy in both eyes with sparing of the central fovea on the right side. In the left macula, there was a subfoveal elevated graywhite membrane with an adjacent punctate area of subretinal hemorrhage (Figure 1). Spectral domain optical coherence tomography (SD-OCT) (Figure 2) and fluorescein angiography (FA) (Figure 3) revealed a subretinal membrane with late staining and mild leakage. The findings were consistent with a subretinal neovascular membrane with mild activity. Outside the central macula, the choriocapillaris and RPE were diffusely absent in both eyes. Given his significant decrease in visual acuity and associated neovascular lesion, treatment with anti-vascular endothelial growth factor (VEGF) therapy was recommended. A total of ten intravitreal injections of $1.25 \mathrm{mg}$ bevacizumab (Avastin ${ }^{\circledR}$; Genentech, San Francisco, CA, USA) were administered over a 12 month period. Visual acuity at last follow-up was 20/150. Clinical exam appeared stable and SD-OCT revealed mild anatomical improvement (Figure 2). The images are unchanged.

\section{Discussion}

Visual loss secondary to choroideremia is classically related to progressive atrophy of the choriocapillaris, RPE, and photoreceptors that initially involves the peripheral retina

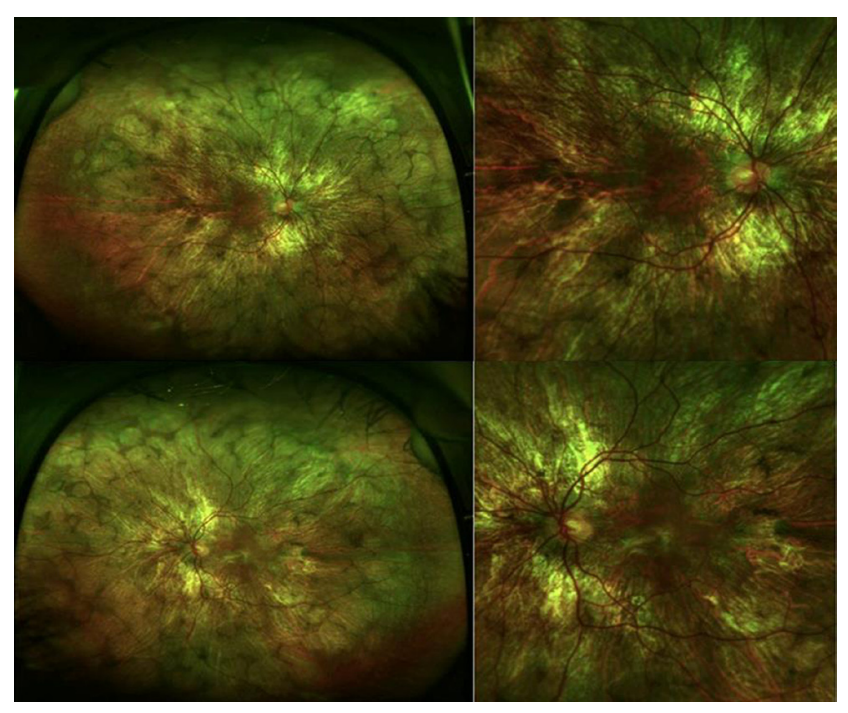

Figure I Ultra-wide field color fundus photograph of the right and left eye depicting diffuse midperipheral chorioretinal atrophy with sparing of the central macula. Note: The left eye also has central submacular fibrosis. and advances centripetally over the course of many decades. A large retrospective study of 115 patients with choroideremia found central visual impairment to be a late manifestation of the disease. The mean age of patients studied was 39 years old. Seventy-nine percent had a visual acuity of 20/40 or better. The majority of patients with severe vision loss (20/200 or worse) were older than 60 years of age. The 5-year rate of vision loss was approximately one line. ${ }^{5}$

Other causes of vision loss in choroideremia include cystoid macular edema (CME) and rarely CNVM. CME may be a relatively common finding in choroideremia with one study identifying cystic changes on SD-OCT in ten of $16(63 \%)$ patients. ${ }^{6} \mathrm{CME}$ is hypothesized to occur secondary to a breakdown in the blood-retina barrier similar to other hereditary chorioretinopathies. Topical dorzolamide showed promise as a potential treatment in a small case series. ${ }^{7}$

In reviewing the literature, five other cases of CNVM in choroideremia have been reported (Table 1). Robinson and Tiedeman ${ }^{8}$ were the first to describe a case in 1987. They presented a 14-year-old boy with known history of choroideremia who developed subacute vision loss in his right eye to 20/200. Clinical examination revealed a grey subretinal membrane with subretinal hemorrhage. Given the subfoveal location of the neovascular net, no treatment was offered. Endo et $\mathrm{al}^{9}$ presented a 17-year-old male and a 46-year-old female, who both developed central subretinal fibrosis secondary to a neovascular membrane. Potter et a ${ }^{10}$ described a similar case in a 27 -year-old female carrier for choroideremia. She presented with unilateral vision loss with an acuity of 20/400, and evidence of subretinal fibrosis on examination. Lastly, Sawa et $\mathrm{al}^{11}$ described a case of bilateral CNVM in a 72-year-old male. This case differed from the others in that it was in an elderly patient who had already suffered diffuse chorioretinal atrophy through the macula.

The pathogenesis of CNVM in choroideremia is presumed to be secondary to degenerative changes of the RPE similar to pathologic myopia. ${ }^{10}$ Pathologic studies of eyes with choroideremia show diffuse atrophy of the choriocapillaris, RPE, and photoreceptors as well as a degenerated Bruch's membrane. Breaks in Bruch's membrane allow for choroidal vessels to grow into the subretinal space. Sawa et $\mathrm{al}^{11}$ also reported the presence of intraretinal neovascularization with angiographic findings resembling retinal angiomatous proliferation.

In most reported cases including the present case, this form of acute vision loss occurs in the second and third decade. This is likely due to a relatively preserved submacular choriocapillaris in this age group. In older patients, progressive atrophy of 
A 2 years before presentation

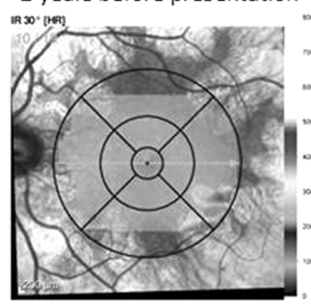

On presentation

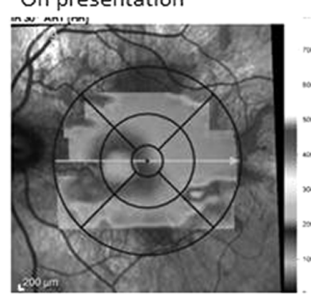

C At last follow-up

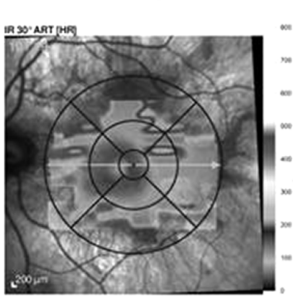

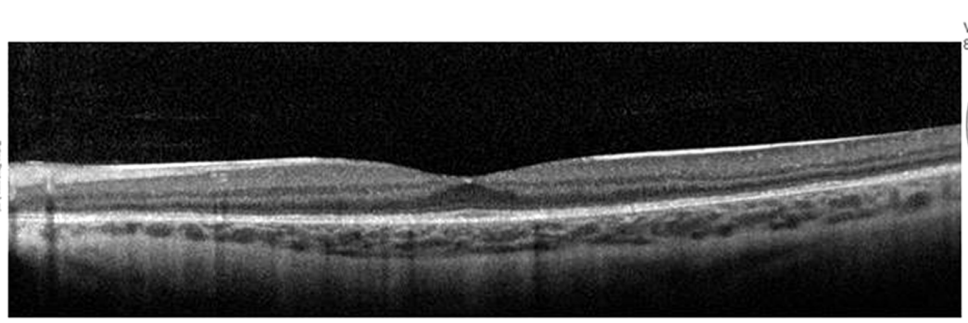

Average thickness [ $\mu \mathrm{m}]$

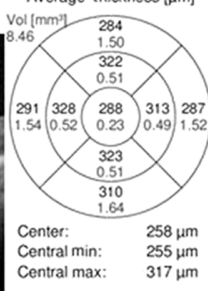

Circle diameters:

, 3, $6 \mathrm{~mm}$ ETDRS

Average thickness [um]

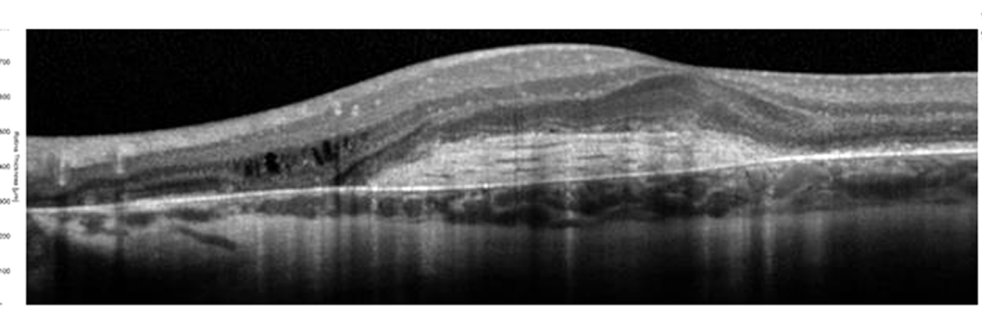

312

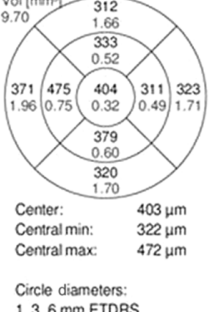

1.3.6 mm ETDRS

Average thickness [um]

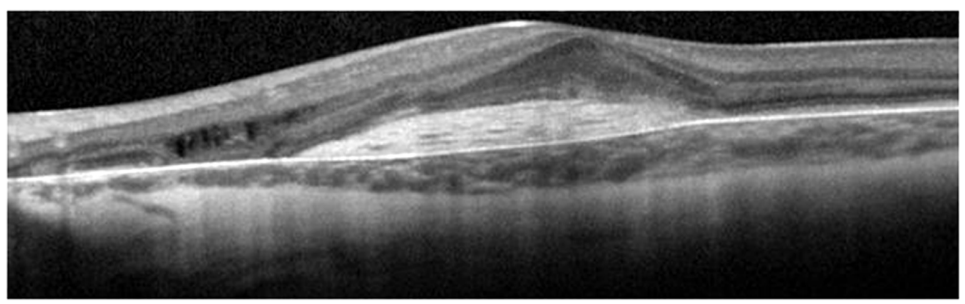

Vol (mmi)

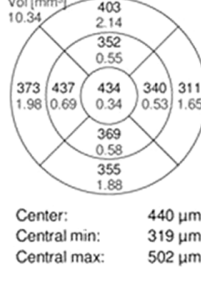

$\begin{array}{ll} & 19 \mu \mathrm{m} \\ \text { Central max: } & 502 \mu \mathrm{m}\end{array}$

Circle diameters:

1.3,6 mm ETDRS

Figure 2 Spectral domain optical coherence tomography horizontal line scan of the left eye prior to development of vision loss, at presentation, and at last follow-up. Notes: Intact outer retinal structures centrally with normal foveal contour and peripheral outer retinal and retinal pigment epithelium atrophy (A). Subretinal hyper-reflective material in the central macula consistent with fibrosis with trace subretinal fluid and mild intraretinal fluid overlying an area of retinal pigment epithelium and choroidal atrophy nasally (B). Mild improvement in nasal intraretinal fluid and subretinal fibrosis with resolution of subretinal fluid after four intravitreal bevacizumab injections (C). Abbreviations: min, minimum; max, maximum; vol, volume; ETDRS, Early Treatment Diabetes Retinopathy Study.

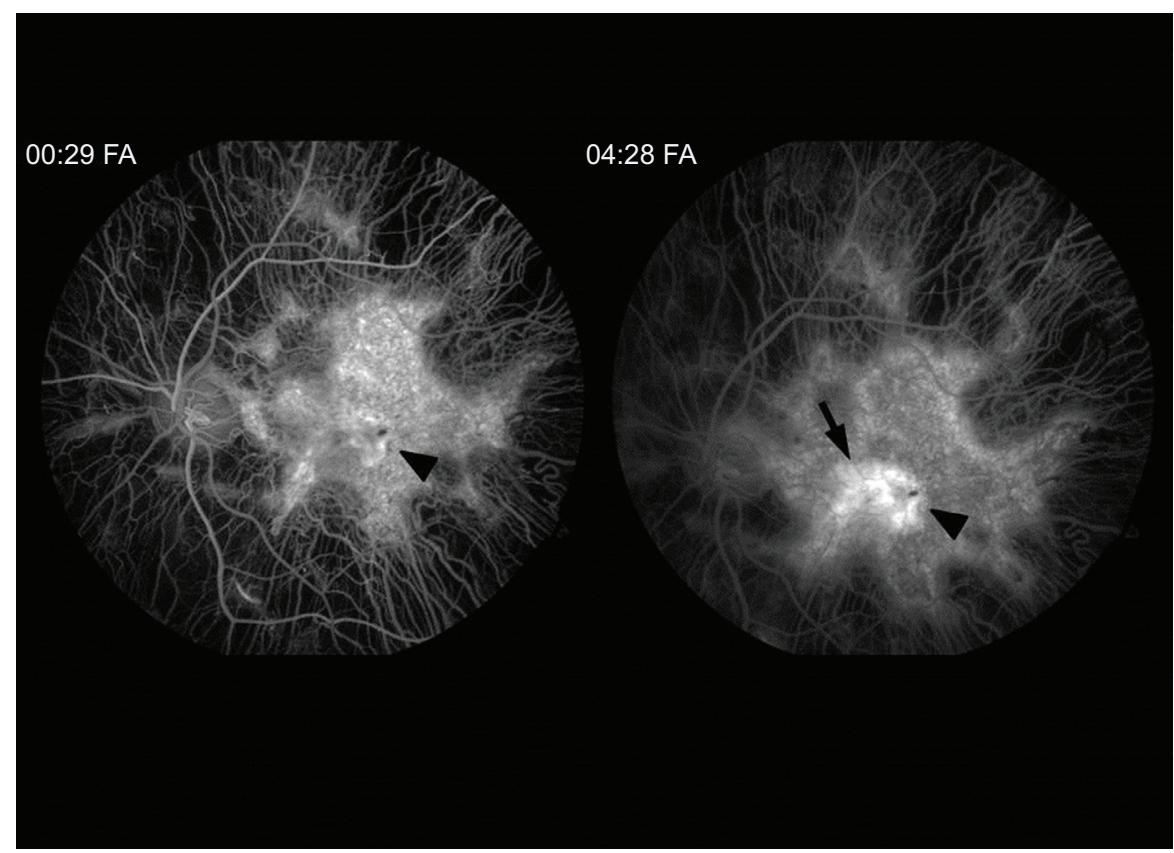

Figure 3 Fluorescein angiogram (FA) transiting the left eye reveals diffuse atrophy of the choriocapillaris sparing the central macula.

Notes: A central hyperfluorescent lesion in the early images stains (00:29) in the late angiographic images centrally (04:28) (arrowhead). Mild leakage is apparent nasally (arrow). 
Table I Presenting features of choroidal neovascularization associated with choroideremia

\begin{tabular}{|c|c|c|c|c|c|c|}
\hline Author year & Age sex & $\begin{array}{l}\text { Presenting } \\
\text { visual acuity }\end{array}$ & Laterality & Location & Fundus features & Intervention \\
\hline $\begin{array}{l}\text { Robinson and Tiedeman } \\
\text { I } 987\end{array}$ & 14 year male & $20 / 200$ & Unilateral & Subfoveal & $\begin{array}{l}\text { Subretinal fibrosis, } \\
\text { subretinal hemorrhage }\end{array}$ & Observation \\
\hline $\begin{array}{l}\text { Endo et al } \\
2000\end{array}$ & 17 year male & $20 / 100$ & Unilateral & Subfoveal & $\begin{array}{l}\text { Subretinal fibrosis, } \\
\text { subretinal hemorrhage } \\
\text { discovered on follow-up }\end{array}$ & Observation \\
\hline & 46 year female & $20 / 28$ & Unilateral & Extrafoveal & Subretinal fibrosis & Observation \\
\hline $\begin{array}{l}\text { Potter et al }{ }^{10} \\
2004\end{array}$ & 27 year female & $20 / 400$ & Unilateral & Subfoveal & Subretinal fibrosis & Observation \\
\hline $\begin{array}{l}\text { Sawa et al"' } \\
2006\end{array}$ & 72 year male & $5 / 400$ & Bilateral & Subfoveal & $\begin{array}{l}\text { Intraretinal } \\
\text { neovascularization }\end{array}$ & Observation \\
\hline $\begin{array}{l}\text { Palejwala et al } \\
2014\end{array}$ & 13 year male & $20 / 150$ & Unilateral & Subfoveal & Subretinal fibrosis & Intravitreal anti-VEGF \\
\hline
\end{tabular}

Abbreviation: VEGF, vascular endothelial growth factor.

the choriocapillaris into the central macula has likely occurred decreasing the risk of formation of a CNVM.

Initial fundus findings include pigmentary changes in the central macula with small subretinal hemorrhage and possible visualization of a subretinal membrane. Significant exudation is rare given that most cases reported show minimal leakage on angiography and paucity of subretinal and intraretinal fluid on OCT.$^{8-11}$ The CNVMs tend to be confined to the area of preserved choriocapillaris. Visual symptoms if present are usually mild and often improve with involution of the lesion. ${ }^{10}$ It has been hypothesized that CNVMs are self-limited because of advancing choriocapillaris and RPE atrophy. ${ }^{9}$ Because of their small size, limited exudation, and propensity for spontaneous involution, CNVM secondary to choroideremia can often go undiagnosed.
Interestingly, of the five reported cases of CNVM associated to choroideremia, two developed in slightly older, 27-year-old, ${ }^{10}$ and 46-year-old, ${ }^{9}$ heterozygous female carriers. Both patients had early "salt and pepper" pattern of RPE atrophy in the mid-periphery without significant choroidal atrophy. Neovascular membranes in the female patients were similar in characteristics to those in males.

Our case had a similar clinical picture to those reported previously and was comprised of significantly reduced visual acuity with small, self-limited, central subretinal fibrosis with minimal exudation. Our case is unique in that anti-VEGF therapy was given to treat the choroideremiaassociated CNVM. The effect of treatment was unfortunately limited by the pre-existing subretinal fibrosis. The visual

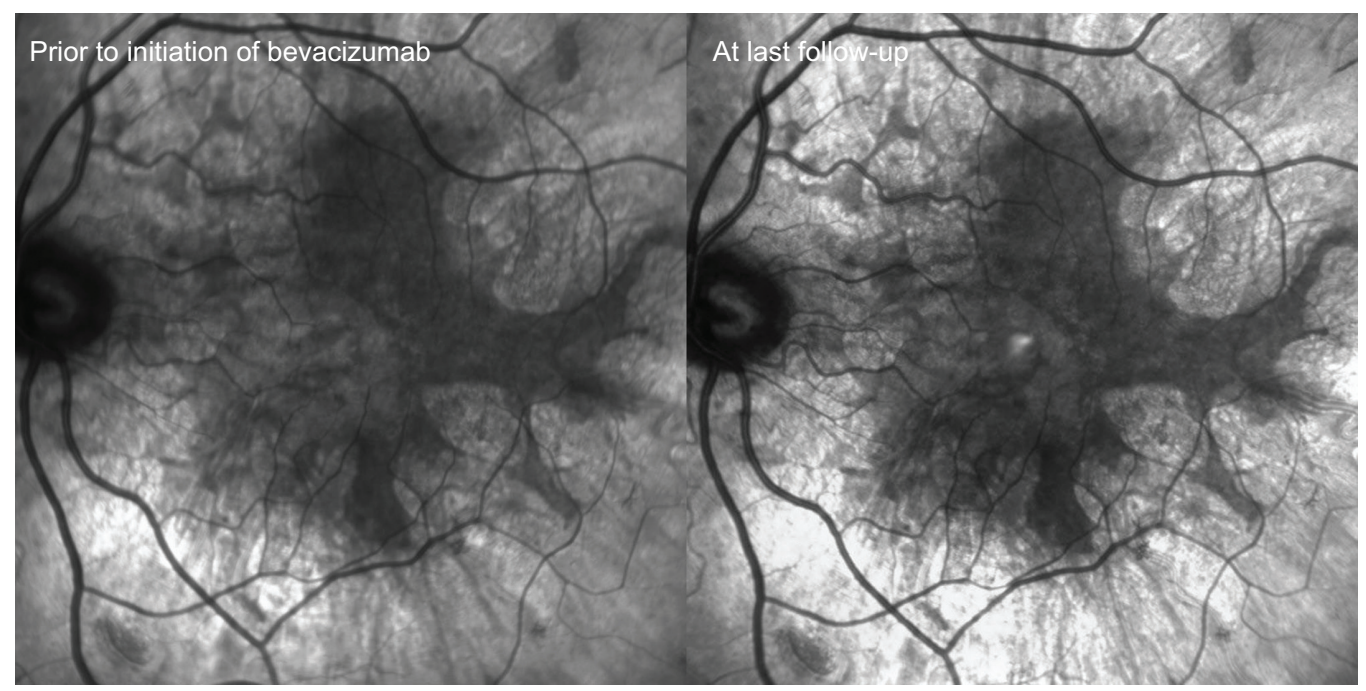

Figure 4 Infrared photos captured prior to initiating anti-vascular endothelial growth factor therapy (bevacizumab) and at last follow-up. Note: Images show no progression of choroidal/retinal pigment epithelium atrophy during the course of treatment. 
acuity remained stable over the course of follow-up. The degradation of the acuity from 20/100 to 20/150 likely represents a fluctuation of visual acuity.

\section{Conclusion}

CNVM in choroideremia is rare and reported cases are in younger patients during the second and third decade before the macula is afflicted by central atrophy. The lesions can be small with minimal leakage and often times spontaneously involute without visual symptoms. Vision loss may not occur until the development of subretinal fibrosis. A trial of anti-VEGF therapy may help reduce CNVM activity, however, after the onset of scarring, visual prognosis is limited.

\section{Acknowledgment}

The authors received an unrestricted grant from Research to Prevent Blindness (New York, NY, USA).

\section{Disclosure}

The authors report no conflicts of interest in this work.

\section{References}

1. Mauthner L. Ein Fall von Choroideremia. Berl Natur-med Ver Innsbruck. 1872;2:191.

2. McCulloch C, McCulloch RJ. A hereditary and clinical study of choroideremia. Trans Am Acad Ophthal Otolaryngol. 1948;52:160-190.

3. Thobani A, Anastasakis A, Fishman GA. Microperimetry and OCT findings in female carriers of choroideremia. Ophthalmic Genet. 2010;31(4):235-239.

4. McCulloch C. Choroideremia: a clinical and pathological review. Trans Am Ophthalmol Soc. 1969;67:142-195.

5. Roberts MF, Fishman GA, Roberts DK, et al. Retrospective, longitudinal, and cross sectional study of visual acuity impairment in choroideraemia. Br J Ophthalmol. 2002;86(6):658-662.

6. Genead MA, Fishman GA. Cystic macular oedema on spectral-domain optical coherence tomography in choroideremia patients without cystic changes on fundus examination. Eye (Lond). 2011;25(1):84-90.

7. Genead MA, McAnany JJ, Fishman GA. Topical dorzolamide for treatment of cystoid macular edema in patients with choroideremia. Retina. 2012;32(4):826-833.

8. Robinson D, Tiedeman J. Choroideremia associated with a subretinal neovascular membrane. Retina. 1987;7(2):70-74.

9. Endo K, Yuzawa M, Ohba N. Choroideremia associated with subretinal neovascular membrane. Acta Ophthalmol Scand. 2000;78(4): 483-486.

10. Potter MJ, Wong E, Szabo SM, McTaggart KE. Clinical findings in a carrier of a new mutation in the choroideremia gene. Ophthalmology. 2004;111(10):1905-1909.

11. Sawa M, Tamaki Y, Klancnik JM, Yannuzzi LA. Intraretinal foveal neovascularization in choroideremia. Retina. 2006;26(5):585-588.
Clinical Ophthalmology

\section{Publish your work in this journal}

Clinical Ophthalmology is an international, peer-reviewed journal covering all subspecialties within ophthalmology. Key topics include: Optometry; Visual science; Pharmacology and drug therapy in eye diseases; Basic Sciences; Primary and Secondary eye care; Patient Safety and Quality of Care Improvements. This journal is indexed on

Submit your manuscript here: http://www.dovepress.com/clinical-ophthalmology-journal

\section{Dovepress}

PubMed Central and CAS, and is the official journal of The Society of Clinical Ophthalmology (SCO). The manuscript management system is completely online and includes a very quick and fair peer-review system, which is all easy to use. Visit http://www.dovepress.com/ testimonials.php to read real quotes from published authors. 